\title{
Breast Cancer Diagnosis: Recent Advances
}

Md Zillur Rahman ${ }^{1 *}$

'Department of Pathology

Bangabandhu Sheikh Muijb Medical University (BSMMU) Dhaka, Bangladesh.
*Correspondence to:

Md Zillur Rahman

Professor

Department of Pathology

Bangabandhu Sheikh Mujib Medical University (BSMMU)

Dhaka, Bangladesh.

Mobile : +8801819315116

Email: drzillur@gmail.com

Date of Submission : 16.01 .2021

Date of Acceptance ： 06.02 .2021
In the management of breast cancer, the advancement of targeted chemotherapy has made a great achievement to reduce the cancer death in the recent past. The advancement of IHC (ImmunoHistoChemistry) is a mile-stone in the development of histopathology as well as in the management of invasive breast cancer. ER, PR and HER2 are the three routinely used molecular markers, Those are targets and / or indicators of highly effective therapies against breast cancer in various clinical settings. Breast cancer is the first type of solid cancer to be successfully treated with molecular targeting therapy. The target is being HER2, validated the general prognostic significance of HER2 gene amplification and protein over expression in the absence of anti-HER2 target therapy. The ability of HER2, as a prognostic factor, is to predict response to hormonal and cytotoxic anti-HER2 targeted therapy, transtuzumab and clinical efficacy of targeting it in a wide variety of clinical settings. The American Society of Clinical Oncology and the College of American Pathologists (ASCOCAP) convened an expert panel, published a guideline in early 2007 and developed recommendations for optimal HER2 testing performance, to improve the accuracy of HER2 testing in invasive breast cancer and its utility as a predictive marker. The updated committee of ASCO-CAP in 2013 recommended that HER-2 status (ie. HER-2 negative or positive) should be determined in all patients with invasive (Early stage or recurrence) breast cancer on the basis of one or more HER2 test results (Negative, equivocal or positive). St Gallen International Expert Consensus on the primary therapy of early breast cancer 2013 recommended a standard duration of adjuvant antiHER2, trastuzumab therapy in HER2 positive patient for one year rather than longer or shorter.

Immunohistochemical stains of breast carcinoma biomarkers are currently performed on patient's biopsy or surgical resections. The use of cytologic samples for determining a patient's ER. PR and HER2 status has yet to be validated. It is commonly determined that the breast cancer patients with ER positive has good prognosis, whereas those with HER-2 positive has a very bad prognosis. But it comes to a difficult situation when we get a triple negative case. Clinically, this heterogenous disease is categorized into three basic therapeutic groups. The ER positive (ER+) group is the most numerous and diverse, with several genomic tests to assist in predicting outcome of ER positive patients receiving endocrine therapy. The HER2 (Also called erbB2) amplified group is a great clinical success because of effective therapeutic targeting of HER2, which has led to intense efforts to characterize other DNA copy number aberrations. Triple Negative Breast Cancers (TNBCs) lacking expression of ER, PR, HER2 also known as basal like breast cancers are a group which lack targeted therapy options and have an increased incidence in patients with germline BRCA1 and TP53 mutations, BRCA1 silencing or of African Ancestry. This is very important to understand the molecular pattern of BRCA1/2 in TNBCs, which are less responsive to targeted therapy. 
Thus, the history of histopathology changed a lot in the diagnosis of breast cancers as well as all other cancers. Routine histopathological diagnosis of breast carcinoma stained with Haematoxylin \& Eosin (H\&E) is just helping us to know the morphological classification, margin clearance and metastasis. But it's of no use in treatment plan and knowing prognosis. We are now more dependent on IHC and progressing to molecular diagnosis for BRCA1 / BRCA2, Sonic Hedgehog (shh) and different other molecules.

Due to lack of facilities and high cost it is hereby advocated for extensive collaboration among the institutes and countries so that recent advances could be available to the common people.

\section{REFERENCES}

1. RC Panguluri et al. Hum Genet. BRCA1 mutations in African Americans. 1999;105(1-2):28-31.

2. Sotiriou C, Wirapati P, Lii S et al. Gene Expression Profiling Breast Cancer :Under standing the molecular basis of Histologic grade to improve Prognosis. J Natl Cancer Inst. 2006;98:262-272.

3. Antonio C Wolff et al. American Society of Clunical Oncology / College of American Pathologists Guideline recommendations for human Epidermal growth factor receptor 2 testing in Breast Cancer. J clin oncol. 2007;25(1):118-145.

4. Filipits M, Rudas M, Jakesz R et al. A New molecular predictor of distant recurrence in ER positive, HER2 negative breast cancer adds indepent information to conventional clinical risk factor. Clin Cancer Res. 2011;17:6012-6020.

5. Hortobagyi GN, Stemmer SM, Burris HA, Yap YS, Sonke GS, Paluch-Shimon S, Campone M, Blackwell KL, André F, Winer EP, Janni W, Verma S, Conte P, Arteaga CL, Cameron DA et al. Ribociclib as firstline therapy for HR positive advanced breast cancer. N Engl J Med. 2016;375:1738-1748.

6. Davies H. Glodzik D, Morganella S et al. HR Detect in a predictor of BRCA1 and BRCA2 deficiency based on mutational signatares. Nat Med. 2017;23:517-525.

7. Ponde N, Brandao M, EL Hachem G et al. Treatment of Advanced HER2 positive breast Cancer, 2018 and beyond. Cancer Treat Rev. 2018;67:10-20

8. Mc Cart Reed AE, Kalaw E, Nones K et al. Phenotypic and molecular dessection of metaplastic Breast Cancer and the Prognostic implication. J Pathol. 2018.

9. Soto-Perez De - Celis E, Loh KP, Baldini C et al. Targeted agents for HER2 positive breast cancer in older adults : Current and future Perspective. Expert opin. Investig Drug S. 2018;27:787-801.

10. Wikins A, Patin E, Harrington K et al. The immunological consequences of rediation induced DNA damage. Pathol. 2019. 